\title{
Nicht auf RAAS-Blocker verzichten
}

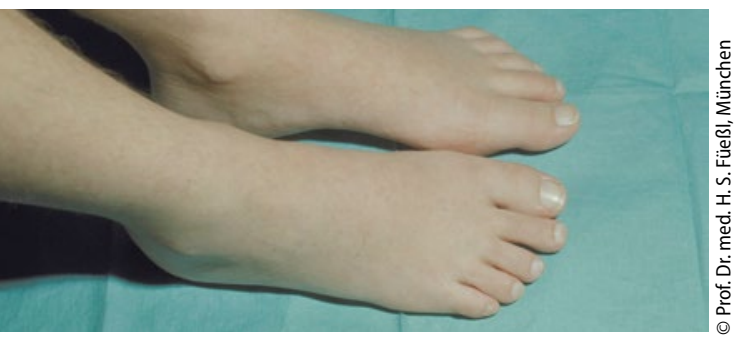

Fußrückenödeme - typische Zeichen einer Niereninsuffizienz.

- Hyperkaliämien sind in der Therapie von Patienten mit Herz- oder Niereninsuffizienz häufig: „Einerseits begünstigen die Erkrankungen selbst die Entstehung erhöhter Kaliumspiegel, andererseits steigern die zur Behandlung dieser Krankheiten eingesetzten Medikamente - und hier insbesondere Hemmer des Renin-Angiotensin-Aldosteron-Systems (RAAS) sowie Aldosteronblocker - die Kaliumwerte zusätzlich “, informierte Prof. Michael Böhm, Homburg/Saar.

\section{RAAS-Blocker: Schlechtere Prognose bei Dosisreduktion}

In der Praxis sind Ärzte daher häufig gezwungen, die Behandlung mit RAASBlockern zu unterbrechen oder die Dosis zu reduzieren. „Das Problemist, dass sich die Prognose der Patienten dann nachweislich sehr rasch verschlechtert", kritisierte Böhm.

Wie Prof. Javed Butler, Stony Brook, NY/USA feststellte, werden RAAS-Blocker im klinischen Alltag oft dauerhaft zu niedrig dosiert. „Es ist dringend nötig, Therapiestrategien zu verfolgen, die eine gute Kontrolle der Kaliumspiegel bei zugleich optimaler RAAS-Blockade ermöglichen“, forderte der Kardiologe. Der Einsatz eines Kaliumbinders könne deshalb einen wichtigen Beitrag zur Op- timierung der Behandlung von Patienten mit Herz- und Niereninsuffizienz leisten, zeigte sich Butler überzeugt.

\section{Oraler Kaliumbinder}

Mit Patiromer wird in Kürze ein neuer, oral zu verabreichender Kationenaustauscher (Kalium gegen Kalzium) für die langfristige Kaliumsenkung verfügbar. In den zulassungsrelevanten klinischen Studien erwies sich Patiromer als effektiv und auch langfristig gut verträglich. „Die Serumkaliumwerte lassen sich unter einer Therapie mit Patiromer so gut kontrollieren, dass auf die wichtige RAASBlocker-Behandlung nicht verzichtet werden muss", schlussfolgerte Böhm. -

Dr. Silke Wedekind

- Satellitensymposium und Experts-on-the-Spot "Enabling and optimizing RAAS therapy in cardio-renal patients by controlling potassium levels", Heart Failure 2017 \& The 4th World Congress on Acute Heart Failure; Paris, Mai 2017 (Veranstalter: Vifor Fresenius Medical Care)

\section{Neue Insulinformulierung}

\section{Nahezu physiologische Wirkung}

_ Der Diabetes mellitus ist einer der entscheidenden Risikofaktoren für ein kardiovaskuläres Ereignis. Dieses Risiko korreliert vorrangig mit der postprandialen Hyperglykämie. „Es besteht eine direkte Beziehung zwischen dem postprandialen Blutzuckerwert und der Progression der KHK“, so Prof. Sebastian Schmidt, Bereichsleiter Endokrinologie, Diabetologie und Stoffwechsel am Universitätsklinikum Lübeck.

Ziel der Forschung ist es, Insulinanaloga zu entwickeln, die dem physiologischen Insulinprofil möglichst nahe kommen. Diesem Anspruch wird das neue Insulin aspart $\left(\right.$ Fiasp $\left.^{\circledR}\right)$ gerecht. Es handelt sich um eine neue, schnell wirksame Formulierung von Insulin aspart (NovoRapid $^{\circledast}$ ), die eine beschleunigte Insulinabsorption und -wirksamkeit bietet.
Fiasp ${ }^{\oplus}$ tritt doppelt so schnell im Blut auf, und innerhalb der ersten 30 Minuten ist die Insulinwirkung um $74 \%$ höher als bei Insulin aspart. Bei Patienten mit einer Insulinpumpe ist die Wirkung innerhalb der ersten 30 Minuten nach Bolusgabe sogar um $100 \%$ gesteigert.

„Vorteilhaft ist die neue, schnell wirksame Insulin-aspart-Formulierung für Patienten, die den Spritz-Ess-Abstand nicht einhalten können, solche mit hohen postprandialen Blutzuckerwerten, fremdbetreute bzw. demente Patienten und Patienten mit vielen Nachkorrekturen bzw. vielen kleinen Mahlzeiten“, so PD Elmar Jaeckel, Hannover.

\section{Dr. Peter Stiefelhagen}

- Satellitensymposium: „Fiasp ${ }^{\circledR}$ - das schnelle Insulin zu den Mahlzeiten im Vergleich zu NovoRapid"," 52. Kongresses der Deutschen Diabetes Gesellschaft; Hamburg, Mai 2017 (Veranstalter: Novo Nordisk)

\section{Funktionelle Verdauungs- störungen pflanzlich lindern}

Das hochdosierte Phytopharmakon Carmenthin ${ }^{\oplus}$ ist eine effektive und gut verträgliche Therapieoption bei funktionellen Magen-Darm-Beschwerden, insbesondere bei leichten Krämpfen, Blähungen und Völlegefühl. Die pharmakologisch wirksamen Inhaltsstoffe wirken synergistisch: Pro magensaftresistenter Weichkapsel sind 90 mg Pfefferminzöl und $50 \mathrm{mg}$ Kümmelöl enthalten. „Pfefferminzöl wirkt direkt an den Schleimhautzellen des Darms" erläuterte Prof. Matthias Enge, Erlangen, beim DGIM-Kongress in Mannheim. Zudem hemme Pfefferminzöl die spannungsabhängigen Kalziumkanäle der glatten Darm-Muskulatur, wodurch es zu krampflösenden Effekten komme. Zusammen mit der schaumhemmenden Potenz des Phytopharmakons ergebe sich ein günstiger Einfluss auf die viszerale Hypersensitivität.

Red. 